\title{
Lattice gauge theories and the Heisenberg antiferromagnetic chain *
}

\author{
F. Berruto, E. Coletti, G. Grignani and P. Sodano \\ Dipartimento di Fisica and Sezione I.N.F.N., \\ Universitá di Perugia, Via Pascoli I-06123 Perugia, Italy
}

We study the strongly coupled 2-flavor lattice Schwinger model and the $S U(2)$-color $Q C D_{2}$. The strong coupling limit, even with its inherent nonuniversality, makes accurate predictions of the spectrum of the continuum models and provides an intuitive picture of the gauge theory vacuum. The massive excitations of the gauge model are computable in terms of spin-spin correlators of the quantum Heisenberg antiferromagnetic spin-1/2 chain.

\section{Introduction}

It is by now well known that, for lattice gauge theories, features of the strong coupling limit have analogs in quantum spin systems. In many cases, the problem of finding the vacuum of the strongly coupled lattice gauge theory is equivalent to finding the ground state of a generalized quantum antiferromagnet [1].

Strong coupling limits of gauge theories are highly non-universal: there are many choices of strong coupling theory which produce identical continuum physics. In spite of this difficulty, there exist strong coupling computations which claim some degree of success [2]. In this contribution we review the results of the ground state and of the spectrum of the strongly coupled 2flavor lattice Schwinger model and of $S U(2)$-color $Q C D_{2}$.

We find that in order to determine the strong coupling vacuum, one has to solve for the ground state of the Heisenberg antiferromagnetic spin chain, and that relevant quantities in the strong coupling expansion can be expressed in terms of spin-spin correlators of the quantum antiferromagnet.

\section{The 2-flavor Schwinger model}

The Hamiltonian and gauge constraint of the continuum 2-flavor Schwinger model are

$H=\int d x\left[\frac{e^{2}}{2} E^{2}(x)+\psi_{a}^{\dagger}(x) \alpha\left(i \partial_{x}+e A(x)\right) \psi_{a}(x)\right]$

*Presented by G. Grignani at Lattice '99, Pisa, Italy. $\partial_{x} E(x)+\psi_{a}^{\dagger}(x) \psi_{a}(x) \sim 0$

with the flavor index $a$ taking the values 1,2 ; there is summation over repeated indices. A lattice Hamiltonian reducing to (1) in the naive continuum limit is $H=H_{0}+\epsilon H_{h}$ with

$$
\begin{aligned}
& H_{0}=\sum_{x=1}^{N} E_{x}^{2} \\
& H_{h}=-i(R-L)
\end{aligned}
$$

and $\epsilon=t / e^{2} a^{2}$. In Eq.(4) the right $R$ and left $L$ hopping operators $\left(L=R^{\dagger}\right)$ are

$R=\sum_{x=1}^{N} R_{x}=\sum_{x=1}^{N} \sum_{a=1}^{2} R_{x}^{(a)}=\sum_{x=1}^{N} \sum_{a=1}^{2} \psi_{a, x+1}^{\dagger} e^{i A} \psi_{a, x}$

The lattice Gauss law constraint is given by

$E_{x}-E_{x-1}+\psi_{1, x}^{\dagger} \psi_{1, x}+\psi_{2, x}^{\dagger} \psi_{2, x}-1 \sim 0$,

where the properly defined charge density reads $\rho(x)=\psi_{1, x}^{\dagger} \psi_{1, x}+\psi_{2, x}^{\dagger} \psi_{2, x}-1$ and vanishes on every site occupied by only one particle.

In a strong coupling perturbative expansion $H_{0}$ is the unperturbed Hamiltonian and $H_{h}$ the perturbation; the ground state of $H_{0}$ is highly degenerate since each state with one particle per site has zero energy [2]. There are $2^{N}$ states of this type. First order perturbations to the vacuum energy vanish. The leading term in the vacuum energy is of order $\epsilon^{2}$

$E_{0}^{(2)}=<H_{h}^{\dagger} \frac{\Pi}{E_{0}^{(0)}-H_{0}} H_{h}>$, 
where the expectation values are defined on the degenerate subspace and $\Pi$ is a projection operator orthogonal to the set of states with one particle per site. Since the charge density on the ground states of $H_{0}$ vanishes, one has that $\left[H_{0}, H_{h}\right]=H_{h}$ holds on any linear combination of the degenerate ground states. Consequently, from Eq.(6), one finds

$E_{0}^{(2)}=-2<R L>\quad$.

Introducing the Schwinger spin operators $\vec{S}_{x}=$ $\psi_{a, x}^{\dagger} \frac{\vec{\sigma}_{a b}}{2} \psi_{b, x}$ and taking into account that the Heisenberg Hamiltonian is $H_{J}=\sum_{x=1}^{N}\left(\vec{S}_{x}\right.$. $\vec{S}_{x+1}-\frac{1}{4}$ ), on the degenerate subspace one has

$$
<H_{J}>=<\sum_{x=1}^{N}\left(-\frac{1}{2} L_{x} R_{x}\right)>\quad
$$

so that

$E_{0}^{(2)}=4<H_{J}>$.

Finding the correct ground state amounts to the diagonalization of the Heisenberg spin Hamiltonian which is exactly diagonalizable in one dimension [3]. On a given site, the presence of a flavor 1 particle can be represented, in the spin model, by the presence of a spin up, a flavor 2 particle by the presence of a spin down. The number of the $H_{J}$ eigenstates is $2^{N}$. Among these, the spin singlet with lowest energy is the non degenerate ground state $\mid$ g.s. $>$. Translational invariance of $\mid$ g.s. $>$ amounts to the invariance of the gauge theory under the discrete chiral symmetry. The eigenvalue of the Heisenberg Hamiltonian on this state in the thermodynamic limit is given by $H_{J} \mid$ g.s. $>=(-N \ln 2) \mid$ g.s. $>$ : due to Eq.(9) the second order correction to the vacuum energy is $E_{g . s .}^{(2)}=-4 N \ln 2$.

There are two different types of excitations which can be created from $\mid$ g.s. $>$. Those that involve only spin flipping and those that involve fermion transport besides spin flipping. The excitations of the first type have lower energy since no electric flux is created, those of the second type have a higher energy. The lowest energy ones occur when the fermion is transported a minimal distance, since the energy is proportional to the coupling times the length of the electric flux that is created. Only the first type of excitations can be described in terms of the Heisenberg model excited states.

In Ref 22 we showed that it is possible to identify the low lying excitations of the Schwinger model with those of the Heisenberg model and that the mass gaps of any other excitation can be expressed as functions of v.e.v.'s of powers of $H_{J}$ and spin-spin correlation functions. We refer to 2] for a detailed analysis of the spectrum and of the chiral symmetry breaking pattern.

\section{The 2-color $Q C D_{2}$}

The Hamiltonian and gauge constraint of the continuum $S U(2)$ color $Q C D_{2}$ are

$$
\begin{aligned}
H & =\int d x\left[\frac{e^{2}}{2} E^{a}(x)^{2}-i \psi_{j}^{\dagger}(x) \alpha \partial_{x} \psi_{j}(x)\right. \\
& \left.-\frac{g}{2} A^{a}(x) \psi_{j}^{\dagger}(x) \alpha \sigma_{j k}^{a} \psi_{k}(x)\right] \\
\partial_{x} E^{a}(x) & +\epsilon^{a b c} E^{b}(x) A^{c}(x)+\frac{1}{2} \psi_{j}(x)^{\dagger} \sigma_{j k}^{a} \psi_{k}(x) \sim 0
\end{aligned}
$$

with $a, b, c=1,2,3, j, k=1,2$; there is summation over repeated indices. The lattice Hamiltonian is $H=H_{0}+\epsilon H_{h}$ with

$H_{0}=\sum_{x=1}^{N} \sum_{a=1}^{3}\left(E_{x}^{a}\right)^{2}, \quad H_{h}=-i(R-L)$

and $\epsilon=t / g^{2} a^{2}$. In Eq.(10) the right $R$ and left $L$ hopping operators $\left(L=R^{\dagger}\right)$ are

$R=\sum_{x=1}^{N} R_{x}=\sum_{x=1}^{N} \sum_{j, k=1}^{2} \psi_{j, x+1}^{\dagger}\left(e^{i \frac{\vec{b} \cdot \vec{A}_{x}}{2}}\right)_{j k} \psi_{k, x}$

The lattice Gauss law constraint reads

$E_{x}^{a}-e^{-i \frac{\vec{\sigma} \cdot \vec{A}_{x-1}}{2}} E_{x-1}^{a} e^{i \frac{\vec{\sigma} \cdot \vec{A}_{x-1}}{2}}+\psi_{j, x}^{\dagger} \frac{\sigma_{j k}^{a}}{2} \psi_{k, x} \sim 0$,

and the charge density is given by $\rho_{x}=\psi_{1, x}^{\dagger} \psi_{1, x}+$ $\psi_{2, x}^{\dagger} \psi_{2, x}-1$.

The ground state of $H_{0}$ is highly degenerate and the degeneracy equals $2^{N}$ since each site is either empty or occupied by two particles in a color singlet. First order perturbations to the vacuum energy vanish and the leading term of the vacuum 
energy is of order $\epsilon^{2}$ and it is given by Eq.(6) with $H_{0}$ and $H_{h}$ obtained from Eqs.(10,10) and $\Pi$ is the projection operator orthogonal to the degeneracy subspace.

If one denotes by $C_{2}$ the quadratic Casimir in the fundamental representation of $S U(2)$, the equation $\left[H_{0}, H_{h}\right]=C_{2} H_{h}$ holds on any linear combination of the degenerate ground states. Following the same steps of section 2 and taking into account that the Schwinger spin operator is now given by $\vec{S}_{x}=\Psi_{x}^{\dagger} \frac{\vec{\sigma}}{2} \Psi_{x}$ where $\Psi=\left(\begin{array}{c}\psi_{1} \\ \psi_{2}^{\dagger}\end{array}\right)$, one gets

$E_{0}^{(2)}=\frac{4}{C_{2}}\left\langle H_{J}\right\rangle=\frac{16}{3}\left\langle H_{J}\right\rangle$

since $C_{2}=3 / 4$.

There are again two types of excitations created from the ground state: those involving only spin flipping (baryons) and those involving fermion transport besides spin flipping (mesons). Of course, the ones which do not involve charge transport have lower energy than the others. As we shall see shortly, the meson masses are computable in terms of spin-spin correlators of the Heisenberg Hamiltonian while baryons are the massless spinons of the spin- $1 / 2$ Heisenberg Hamiltonian.

The lowest lying massive excitations are a pseudoscalar and a scalar meson created by the Fourier transform of the conserved gauge invariant currents at zero momentum $\sum_{x} j_{1}(x)=R+L$ and $\sum_{x} j_{5}(x)=R-L$, respectively; namely,

$|P\rangle=(R+L)|g . s\rangle,. \quad|S\rangle=(R-L)|g . s$.

At the zero-th order they are degenerate, but the degeneracy is removed at the second order in the strong coupling expansion. The mass of these excitations is obtained by computing their energies and by subtracting the ground state energy. Up to the second order in $\epsilon$, the mass of the state $|P\rangle$ is given by [4]

$$
\begin{gathered}
m_{P}=\frac{g^{2} a}{2}\left(\frac{3}{4}-\epsilon^{2} \frac{20\left\langle\sum_{x=1}^{N} \vec{S}_{x} \cdot \vec{S}_{x+2}-\frac{1}{4}\right\rangle+16\left\langle H_{J}\right\rangle}{3 N+3\left\langle H_{J}\right\rangle}\right) \\
=\frac{g^{2} a}{2}\left(\frac{3}{4}+\epsilon^{2} 54.10\right),
\end{gathered}
$$

whereas the one of the scalar meson $|S\rangle$ is

$$
m_{S}=\frac{g^{2} a}{2}\left(\frac{3}{4}-\epsilon^{2} \frac{32\left\langle\sum_{x=1}^{N} \vec{S}_{x} \cdot \vec{S}_{x+2}-\frac{1}{4}\right\rangle-128\left\langle H_{J}\right\rangle}{2\left\langle H_{J}\right\rangle}\right)
$$

$$
=\frac{g^{2} a}{2}\left(\frac{3}{4}+\epsilon^{2} 62.43\right) \text {. }
$$

\section{Concluding remarks}

We have shown how, in the strong coupling limit, all the massive low lying excitations of some gauge models can be computed in terms of spinspin correlators of the Heisenberg model and that the massless excitations correspond to the spinons of the quantum antiferromagnet. As evidenced in [5], the explicit evaluation of the pertinent spinspin correlators is far from being trivial and thus evaluating the spectrum in higher orders of strong coupling perturbative expansion may be quite involved. It is comforting to observe that, already at the second order in the strong coupling expansion, one gets results in satisfactory agreement with the continuum theory [2]. For the $S U(2)-$ color $Q C D_{2}$, setting $t=1$, one gets $m_{P}=1.093$, which is $37 \%$ higher than the result obtained in [6] in the continuum for $\mathcal{N}_{c}=2$ and is consistent with the lattice numerical calculations of ref.[7]. For $m_{S}$ one gets $m_{S}=1.133$, this mass has not been computed in the continuum.

\section{REFERENCES}

1. J. Smit, Nucl Phys. B175 307 (1980); G. W. Semenoff, Mod. Phys. Lett, A7, 2811 (1992); E. Langmann and G. W. Semenoff, Phys. Lett. B297, 175 (1992); M. C. Diamantini, E. Langman, G. W. Semenoff and P. Sodano, Nucl. Phys. B406, 595 (1993).

2. F. Berruto, G. Grignani, G. W. Semenoff and P. Sodano, Phys. Rev. D57 (1998) 5070; ibid. D59, 034504 (1999); Ann. of Phys. 275, 254 (1999) and refs. therein.

3. H. Bethe, Z. Physik 71, 205 (1931).

4. E. Coletti, Teorie di gauge e modelli di spin, Master Thesis, University of Perugia, 1999.

5. V. E. Korepin, A. G. Izergin, F. H. L. Essler and D. B. Uglov, Phys. Lett. A190, 182 (1994);I. Affleck, J. Phys. A31, 4573 (1998).

6. G. Bhattacharya, Nucl. Phys. B205, 461 (1982) .

7. J-Q. Jiang, X-Q. Luo, Z-H. Mei, H. Jirari, H. Kröger and C-M. Wu, Phys. Rev. D60, 014501 (1999). 